\title{
Cloning, production and characterization of a glycoside hydrolase family 7 enzyme from the gut microbiota of the termite Coptotermes curvignathus
}

\begin{abstract}
Coptotermes curvignathus is a termite that, owing to its ability to digest living trees, serves as a gold mine for robust industrial enzymes. This unique characteristic reflects the presence of very efficient hydrolytic enzyme systems including cellulases. Transcriptomic analyses of the gut of C. curvignathus revealed that carbohydrate-active enzymes (CAZy) were encoded by 3254 transcripts and that included 69 transcripts encoding glycoside hydrolase family 7 (GHF7) enzymes. Since GHF7 enzymes are useful to the biomass conversion industry, a gene encoding for a GHF7 enzyme (Gh1254) was synthesized, sub-cloned and expressed in the methylotrophic yeast Pichia pastoris. Expressed GH1254 had an apparent molecular mass of $42 \mathrm{kDa}$, but purification was hampered by its low expression levels in shaken flasks. To obtain more of the enzyme, GH1254 was produced in a bioreactor that resulted in a fourfold increase in crude enzyme levels. The purified enzyme was active towards soluble synthetic substrates such as 4-methylumbelliferyl- $\beta$-d-cellobioside, 4-nitrophenyl- $\beta$-d-cellobioside and 4-nitrophenyl- $\beta$-d-lactoside but was non-hydrolytic towards Avicel or carboxymethyl cellulose. GH1254 catalyzed optimally at $35{ }^{\circ} \mathrm{C}$ and maintained $70 \%$ of its activity at $25{ }^{\circ} \mathrm{C}$. This enzyme is thus potentially useful in food industries employing low-temperature conditions.
\end{abstract}

Keyword: Avicel; Biomass; Bioreactor; Cellulase; Saccharification; Symbiont 